\title{
MULTIPLE SPATIAL SCALES IN ENGINEERING AND ATMOSPHERIC LOW MACH NUMBER FLOWS
}

\author{
RUPERT KLEIN ${ }^{1}$
}

\begin{abstract}
The first part of this paper reviews the single time scale/multiple length scale low Mach number asymptotic analysis by Klein $(1995,2004)$. This theory explicitly reveals the interaction of small scale, quasi-incompressible variable density flows with long wave linear acoustic modes through baroclinic vorticity generation and asymptotic accumulation of large scale energy fluxes. The theory is motivated by examples from thermoacoustics and combustion. In an almost obvious way specializations of this theory to a single spatial scale reproduce automatically the zero Mach number variable density flow equations for the small scales, and the linear acoustic equations with spatially varying speed of sound for the large scales. Following the same line of thought we show how a large number of wellknown simplified equations of theoretical meteorology can be derived in a unified fashion directly from the three-dimensional compressible flow equations through systematic (low Mach number) asymptotics. Atmospheric flows are, however, characterized by several singular perturbation parameters that appear in addition to the Mach number, and that are defined independently of any particular length or time scale associated with some specific flow phenomenon. These are the ratio of the centripetal acceleration due to the earth's rotation vs. the acceleration of gravity, and the ratio of the sound speed $v s$. the rotational velocity of points on the equator. To systematically incorporate these parameters in an asymptotic approach, we couple them with the square root of the Mach number in a particular distinguished so that we are left with a single small asymptotic expansion parameter, $\varepsilon$. Of course, more familiar parameters, such as the Rossby and Froude numbers may then be expressed in terms of $\varepsilon$ as well. Next we consider a very general asymptotic ansatz involving multiple horizontal and vertical as well as multiple time scales. Various restrictions of the general ansatz to only one horizontal, one vertical, and one time scale lead directly to the family of simplified model equations mentioned above. Of course, the main purpose of the general multiple scales ansatz is to provide the means to derive true multiscale models which describe interactions between the various phenomena described by the members of the simplified model family. In this context we will summarize a recent systematic development of multiscale models for the tropics (with Majda).
\end{abstract}

Mathematics Subject Classification. 34E13, 76B99, 76Q05, 86A10.

Plenary lecture, Low Mach Number Flows Conference, June 21-25, 2004, Porquerolles, France.

\section{INTRODUCTION}

Two classes of simplified model equations are typically derived through low Mach number asymptotics. These are zero Mach number models describing quasi-incompressible, variable density flows on the one hand, see, e.g., [23], and linearized and weakly nonlinear acoustic theories on the other, see, e.g., [34,39] and the

Keywords and phrases. Low Mach number flows, multiple scales asymptotics, atmospheric flows.

${ }^{1}$ FB Mathematik \& Informatik, Freie Universität Berlin, Zuse Institut, Takustr. 7, 14195 Berlin, Germany.

(c) EDP Sciences, SMAI 2005 
references therein. In this context, acoustic modes are often thought of as "high-frequency modes" evolving on time scales much shorter than that of advection. Whereas the derivation of zero Mach number models proceeds via "standard" single time/single length scale analyses, the analysis of high frequency acoustics requires multiple time scale asymptotics.

Of comparable interest in applications, but represented much less prominently in the literature, are problems in which long wave acoustic modes interact with advection on a common time scale. This is motivated by the physics of thermoacoustic refrigeration and of accelerating premixed flames in the first part of this paper. Then we review the single time scale/multiple length scale low Mach number asymptotic analysis from [12,15], which explicitly reveals the mathematics of this interaction: in the resulting multiscale model the zero Mach number variable density flow equations describe the small scale motions, linear acoustics represents the long waves, and there are specific coupling terms involving correlations between the small scale density and velocity fluctuations. In turn it follows that in the absence of small-scale leading order density variations the large scale linear acoustics entirely decouple from the small scale flow, at least on the time scales considered here. A rigorous proof for the decoupling in this regime is reported on by Schochet elsewhere in this volume.

One almost trivial aspect of this multiple scales theory has motivated the author's recent effort at constructing a unified mathematical approach to meteorological modelling [13-15]: when restricted to the small scales only, the theory reproduces the zero Mach number variable density flow equations. When restricted to the long wavelengths only, it reproduces the linear acoustic equations with spatially varying speed of sound.

It turns out that a large number of well-known simplified model equations of theoretical meteorology can be derived in a unified fashion directly from the three-dimensional compressible flow equations through just this mechanism. A preparatory step is the introduction of a particular distinguished limit among, effectively, the Mach, Froude, Rossby, and other characteristic dimensionless numbers. This leaves us with a single small asymptotic expansion parameter, $\varepsilon$. In a second step we introduce a very general asymptotic ansatz involving multiple horizontal and vertical as well as multiple time scales. Next, various different restrictions of the general ansatz to only one horizontal, one vertical, and one time scale (plus some standard asymptotic arguments) lead directly to the family of simplified model equations mentioned above.

Up to now only a single one of the well established meteorological model equations studied by the author turned out not to fit this scheme straight-forwardly. These are the so-called Hydrostatic Primitive Equations (HPEs) which form the basis of many weather prediction codes and climate models today. The HPEs assume order $O(1)$ Mach number for the horizontal flow so as to ensure that the model captures the (weak) compressibility effects on large scales that are being observed in the form of Lamb-waves [28]. Also, local flow velocities within high tropospheric jets can reach Mach numbers of the order $\mathrm{M}=0.2 \ldots 0.3$ which further motivates the assumption of Mach numbers of order $O(1)$ at least for horizontal motions. In this paper we show for the first time how the HPEs can also be derived within our general multiscale asymptotic framework.

Of course, besides providing a systematic approach to the derivation of existing single scale models (one horizontal, one vertical, and one characteristic time scale) the general multiple scales ansatz gives rise to new true multiscale models. These capture interactions between the various phenomena that are otherwise described by different single scale models. In this context we will summarize recent systematic developments of multiscale models for the tropics (with Majda).

The rest of this paper is organized as follows. Section 1 considers low Mach number flows in the classical sense, ignoring singular effects of rotation and gravity. Sections 1.1, 1.2 motivate the analysis by briefly discussing the physics of a thermoacoustic refrigerator and of accelerating premixed flames in large scale enclosures. Section 1.3 introduces the single time - multiple length scale asymptotic ansatz for vanishing Mach number. Section 1.4 demonstrates how single scale specializations of the ansatz reproduce linear acoustics on the large scales and the equations of incompressible, variable density flow for the small scales. Section 1.5 shows that there are nontrivial interactions between the small and large scales in the presence of small scale, leading order density variations.

In Section 2 we then consider atmospheric flows. First Section 2.1 discusses the so-called "anelastic constraint" and its variants, which replace the zero velocity divergence condition from zero Mach number theory for flows 
covering a typical atmospheric pressure scale height in the vertical direction. Section 2.2 demonstrates that there are (at least) three non-dimensional characteristic parameters for atmospheric flows that are independent of the length and time scales of any specific phenomenon or flow regime. That is, they are determined solely by some globally given determining quantities, such as the gravitational acceleration, the earth's radius and rotation rate, the total mass of the atmosphere, and the global radiation balance. One of these parameters is of order $O(1)$, while two of them are small, giving rise to asymptotic considerations. In our view, it is the existence of such globally valid singular perturbation parameters which justifies a systematic search for simplified models based on various length and time scaling assumptions! Accordingly, we propose a particular distinguished limit among the two small parameters and proceed with multiple scales asymptotics in terms of the remaining small parameter in the sequel. Section 2.3 describes the unified approach to meteorological modelling proposed in [13-15] and demonstrates how it allows one to recover various simplified models of theoretical meteorology directly from the full compressible flow equations. The examples discussed are Mesoscale anelastic and buoyancycontrolled (weak temperature gradient, WTG) models, the Quasi-Geostrophic Theory, and - for the first time in this framework - the Hydrostatic Primitive Equations. Section 2.4 finally discusses the Intraseasonal Planetary Equatorial Synoptic Dynamics model (IPESD), derived originally by Majda and the author in [22], which is a true multiscale application of the proposed general modelling ansatz.

Section 3 summarizes and draws a few conclusions.

\section{LOW MaCh NUMBER FLOWS With MULTiPle SPATIAL SCALES}

\subsection{Thermoacoustic refrigerator}

Consider a long, straight resonance tube, closed at one end and equipped with a loudspeaker at the other. At a distance of about one quarter of the length of the tube away from the closed end there are three narrowly spaced stacks of heat exchanger plates as sketched in Figure 1 (top, lower graph). The two outer stacks will be in contact with the environment and with the object to be cooled, the middle stack is isolated from the environment and functions as a medium for the transport of heat between the cold and the hot heat exchanger stacks.

Assume that a standing acoustic wave with wavelength equal to four times the tube length is being maintained by the loudspeaker (Fig. 1 - top, upper graph), and consider the motion of fluid parcels within the tube (Fig. 1 bottom left, upper graph). Due to velocity perturbations induced by the pressure wave, a parcel will be displaced in an oscillatory fashion and it will undergo pressure and temperature fluctuations. For example, a parcel near the closed end of the tube moves towards the closed end as the medium reaches maximum compression. When the gas expands in the opposite phase of the cycle, the parcels' pressure and temperature drop and it moves away from the closed end. Thus, fluid particles generally trace a local temperature gradient by this oscillatory motion (Fig. 1 - bottom left, lower graph).

If the stack has thin, closely spaced plates, then axial conduction along the plates is minute, while heat exchange between the gas and the plates is intense. Initially, the plates are in thermal equilibrium with the gas, which in turn has constant temperature. A parcel of the gas will, when moving towards the closed end of the tube, become hotter than the plate locally and it will release heat to the plate. As it moves to the left and expands, it becomes colder than the plate and receives heat. The net effect of one oscillatory cycle is to move heat along the plate. Over many cycles, unless the plate is heated, its left end and the surrounding gas are cooled; ultimately, the plates will acquire a temperature distribution which at every point has a gradient similar to that traced by the parcels during their acoustic motion. If heat is added to the left end at some temperature lower than ambient but higher than the plate's long time equilibrium temperature, a persistent heat transport from this low temperature reservoir to the ambient is established.

The flow field within such a device is a perfect example of a single time scale / multiple space scale low Mach number flow. Its characteristic time is the oscillation time of the acoustic standing wave imposed by the loudspeaker. The spatial scales involved are the tube length on the one hand, and the stack spacing on the 


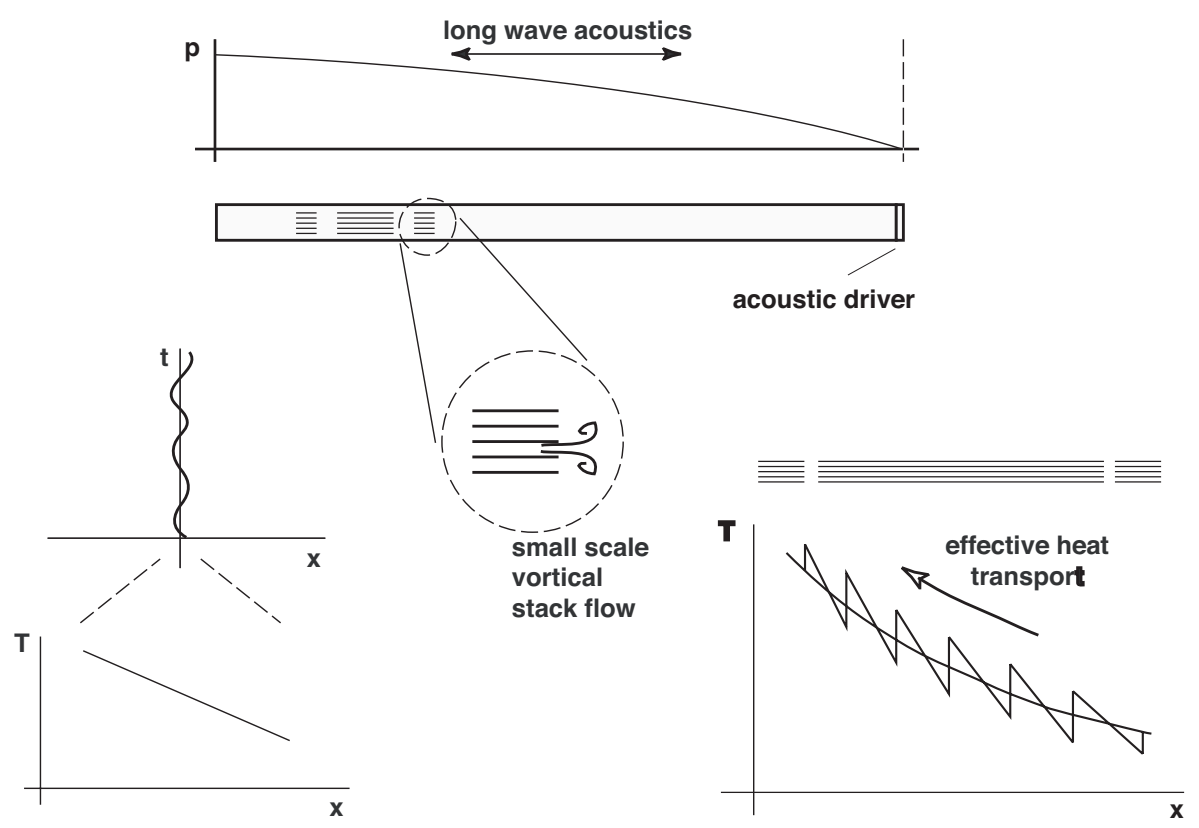

Figure 1. Conceptual sketches illustrating the mechanism enabling thermoacoustic refrigeration. Top left: quarter wavelength acoustic standing wave and resonance tube with heat exchanger stacks. Bottom left: a parcel's motion between the plates in a space-time diagram and the temperature gradient it traces due to the cyclic adiabatic compression and expansion. Bottom right: Mechanism of cold-to-hot heat transfer along the middle stack.

other. Figure 1 (center) indicates, e.g., the small scale strongly vortical flow that will develop near the end of the plate stack.

See [30-33] for discussions of appropriate numerical techniques for the simulation of such thermoacoustic devices and for extensive numerical results.

\subsection{Accelerating flames}

As mentioned, the characteristic time scale in the previous example of a thermoacoustic device is imposed by the long wavelength acoustic mode. Here we consider a different situation. Again we have a single time and multiple spatial scales, but here it will be the small scale motions which impose the evolution time scale. Figure 2 displays a result from a two-dimensional model simulation in [35] of the russian RUT facility [7]. A channel with dimensions $60 \mathrm{~m} \times 2.5 \mathrm{~m} \times 2.0 \mathrm{~m}$ and equipped with a sequence of obstacles has been filled with an explosive, nearly homogeneous hydrogen-air mixture. After ignition, a slowly burning flame kernel establishes as seen in the first panel. The flame burning velocity and the gas velocities induced by the thermal expansion within the flame are of the order of a few meters per second at this stage.

As the burnt gas region has grown sufficiently to fill the entire cross-section of the channel, the induced gas motion becomes directed along the channel axis. As the flame motion is a composition of advection by the surrounding flow and the relative motion between flame and unburnt gas, the flame surface is increasingly extended in the channel direction, and its overall flame surface area increases continuously as seen in the second panel of Figure 2.

From here on, there are two main effects leading to an explosive development of the effective flame speed in time. The first is the increasing level of turbulence in the wakes of the obstacles. As the unburnt gas is pushed through the channel at ever increasing speeds, the generation of turbulence in the wakes of the obstacles 


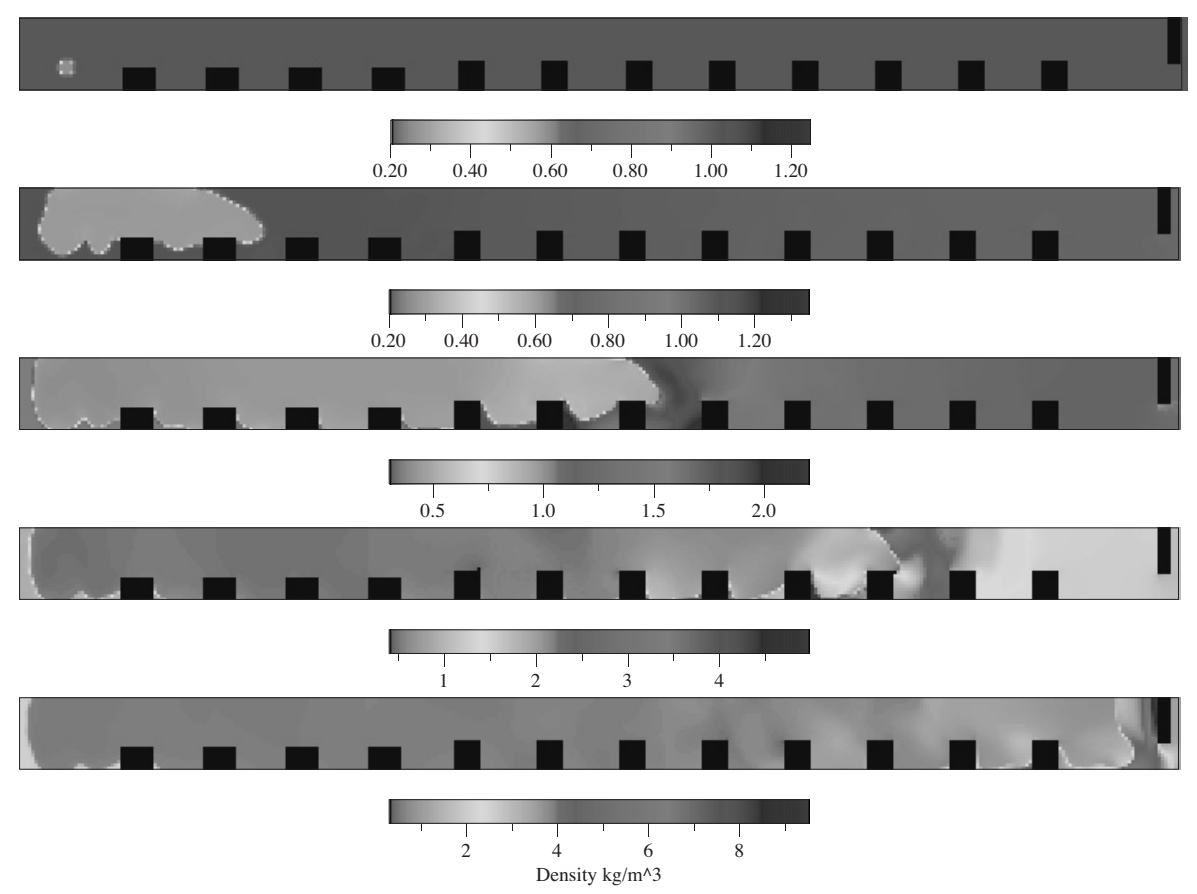

Figure 2. Accelerating flame in a 2D proxy simulation of a large scale explosion event, from [35].

increases accordingly. When the flame reaches these areas of high turbulence intensity, it accelerates. Probably more important is the simple effect of overall flame area increase. Due to the rapid advection of the flame tip along the channel, and the relatively slow lateral motion of the flame in the zones between the obstacles, the total flame area increases roughly in proportion with the distance the flame tip has traveled along the channel. The total flame area, in turn, is responsible for the net volume production due to combustion, and thus for the total volume flux along the channel in front of the flame.

The net result of this acceleration is seen in the space-time-plot of Figure 3, which compares measurements in the RUT-facility with the simulations described above. The absolute propagation speed of the flame front increases from a few meters per second initially to about $750 \mathrm{~m} / \mathrm{s}$ by the time the flame reaches the end of the obstacle array.

It should be mentioned that the "d $t=0.32$ " label in the graph indicates that for the comparison the time axis of the simulation has been shifted by $0.32 \mathrm{~s}$ so as to make the end points of the experimental and simulated space-time traces match. This is reasonable, because the initial phase of the experiment, due to a point ignition of the gas in the experiment, cannot be faithfully represented by the two-dimensional computational model: an ignition- "point" in the computational model corresponds with an entire line of ignition in three dimensions.

The key point of this process, from the point of view of low Mach number analysis, is that the entire flow field is generated by the accelerating flame, so that combustion, advection, and compression wave propagation are all synchronized to the same characteristic time scale. Therefore we have again a typical single time-multiple space scale regime.

\subsection{Single time, multiple space scale expansions for low Mach number flows}

The examples discussed in the previous sections suggest a single time - multiple space scale expansion in terms of the Mach number

$$
\mathrm{M}=\frac{u_{\text {ref }}}{c_{\text {ref }}} \ll 1 .
$$




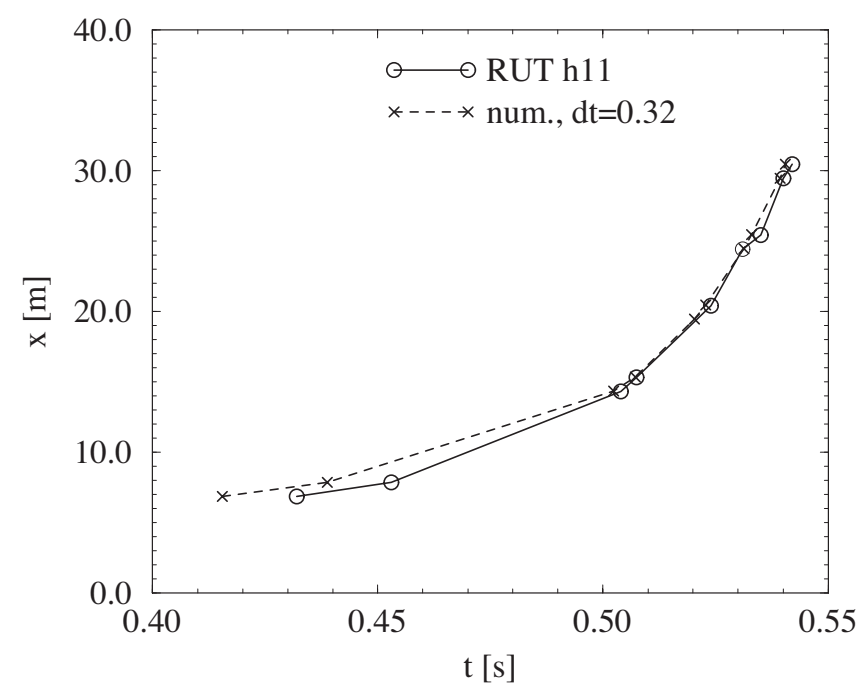

FiguRE 3. Flame acceleration in a space-time diagram and comparison of experimental and numerical results.

For simplicity of exposition we consider here the Euler equations for compressible flow, i.e.,

$$
\begin{aligned}
\rho_{t}+\nabla \cdot(\rho \boldsymbol{v}) & =0, \\
(\rho \boldsymbol{v})_{t}+\nabla \cdot(\rho \boldsymbol{v} \circ \boldsymbol{v})+\frac{1}{\mathrm{M}^{2}} \nabla p & =0, \\
(\rho e)_{t}+\nabla \cdot([\rho e+p] \boldsymbol{v}) & =0 .
\end{aligned}
$$

Here $(\rho, \boldsymbol{v}, p)$ are the density, velocity, and pressure, respectively. The total energy density, $\rho e$, obeys the equation of state,

$$
\rho e=\frac{p}{\gamma-1}+\mathrm{M}^{2} \frac{\rho \boldsymbol{v}^{2}}{2},
$$

with $\gamma$ the isentropic exponent, which is assumed constant here.

Consider a one-dimensional setting as sketched in Figure 4. The graph on the left sketches a situation in which, e.g., an unstable flame drives the surrounding flow field, thereby simultaneously inducing advectively transported fluctuations of entropy, vorticity, and chemical species, and acoustic pressure perturbations. As the time scale, $T$, of the oscillations is imposed by the fluctuating flame, the difference in propagation speeds, $u_{\text {ref }} \ll c_{\text {ref }}$, results in differing characteristic lengths, $L$ and $L / \mathrm{M}$, of the associated advective and acoustic phenomena, respectively. This regime may be captured by a multiple space scale asymptotic expansion of the form [12],

$$
\mathbf{U}(\boldsymbol{x}, t ; \mathrm{M})=\sum_{i} \mathrm{M}^{i} \mathbf{U}^{(i)}(\boldsymbol{x}, \boldsymbol{\xi}, t), \quad \text { where } \quad \boldsymbol{\xi}=\mathrm{M} \boldsymbol{x} .
$$

The opposite regime, sketched in Figure 4b, features a single characteristic length but multiple time scales. For further information on this regime the reader is referred to Schochet's contribution in this volume [34].

The analysis for the compressible Euler equations (2), based on the expansion in (4) straight-forwardly yields the following results for the leading, first, and second order pressure from the momentum equations, due to the fact that to orders $O\left(\mathrm{M}^{-2}\right)$ and $O\left(\mathrm{M}^{-1}\right)$ there is no other term that could balance those resulting from the expansion of the pressure gradient. 


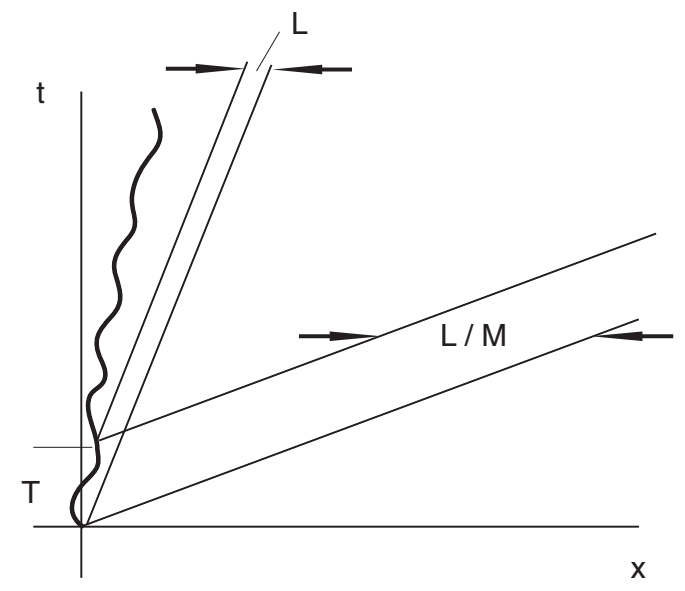

a)

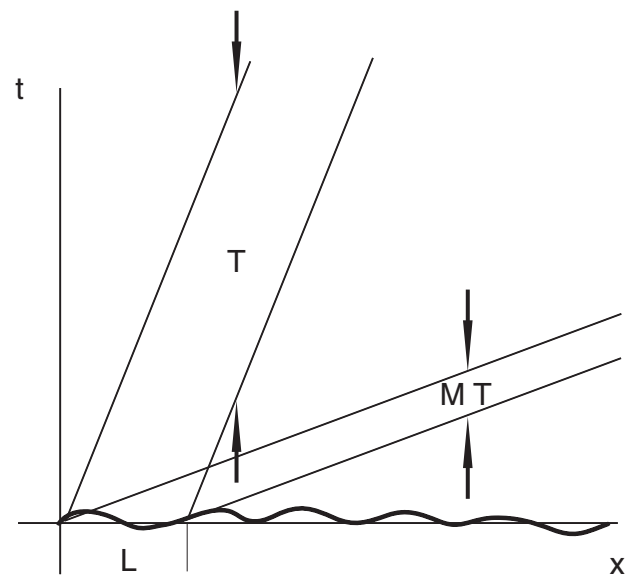

b)

Figure 4. Single time, multiple space scales, (a), vs. multiple time, single space scales, (b), for low Mach number flows. Thick wiggly lines indicate perturbations, imposed locally in an oscillatory fashion, (a), or with given spatial variation at some point in time, (b).

Pressure decomposition

$$
p^{(0)} \equiv P_{0}(t), \quad p^{(1)}=P^{(1)}(\boldsymbol{\xi}, t), \quad p^{(2)}=p^{(2)}(\boldsymbol{x}, \boldsymbol{\xi}, t)
$$

Using (5) 1 and the equation of state in (3) a simplification of the energy equation occurs in that $(\rho e)^{(0)}=P_{0}(t) /(\gamma-1), \nabla(\rho e) \equiv 0$. This justifies equation $(6)_{3}$ below. The leading order mass balance retains the structure of the original equation $(2)_{1}$, except that it is formulated with only the leading order density and velocities, and - except for the expansion of the pressure gradient term - the same holds for the second order momentum equation. Dropping the $(\cdot)^{(0)}$-superscript on $\rho, \boldsymbol{v}$ for convenience of notation we have the equations for

Small scale quasi-incompressible flow:

$$
\begin{aligned}
\rho_{t}+\nabla_{\boldsymbol{x}} \cdot(\rho \boldsymbol{v}) & =0 \\
(\rho \boldsymbol{v})_{t}+\nabla_{\boldsymbol{x}} \cdot(\rho \boldsymbol{v} \circ \boldsymbol{v})+\nabla_{\boldsymbol{x}} p^{(2)} & =-\nabla_{\boldsymbol{\xi}} P^{(1)}, \\
\gamma P_{0} \quad \nabla_{\boldsymbol{x}} \cdot \boldsymbol{v} & =-\frac{\mathrm{d} P_{0}}{\mathrm{~d} t} .
\end{aligned}
$$

Integrating (6) 2 w.r.t. $\boldsymbol{x}$ over some domain $\Omega \supset B_{r}$, with $B_{r} \subset \mathbb{R}^{3}$ a ball of radius $r$, dividing by $|\Omega|$, letting $r \rightarrow \infty$, and requiring that any of perturbation functions, say $\phi^{(i)}$ satisfies the sublinear growth condition

$$
\lim _{\varepsilon \rightarrow 0} \varepsilon \phi^{(i)}\left(\frac{\boldsymbol{\xi}^{\prime}}{\varepsilon}, \boldsymbol{\xi}, t\right)=0 .
$$

For any values of $\left(\xi^{\prime}, \xi\right)$ we obtain the long wave momentum balance in $(8)_{1}$. The same procedure is applied to the first order energy equation (not shown) to obtain $(8)_{2}$, so that we obtain the equations 
for Long wave acoustics:

$$
\begin{aligned}
(\overline{\rho \boldsymbol{v}})_{t}+\nabla_{\boldsymbol{\xi}} P^{(1)} & =0, \\
P_{t}^{(1)}+\gamma P_{0} \nabla_{\boldsymbol{\xi}} \cdot \overline{\boldsymbol{v}} & =0 .
\end{aligned}
$$

Here the overbar denotes averages of the perturbation functions in the small scale variable $\boldsymbol{x}$, i.e.,

$$
\bar{\phi}(\boldsymbol{\xi}, t)=\lim _{\varepsilon \rightarrow 0} \frac{\varepsilon^{d}}{\left|B_{1}\right|} \int_{B_{1}} \phi\left(\frac{\boldsymbol{\xi}^{\prime}}{\varepsilon}, \boldsymbol{\xi}, t\right) \mathrm{d}^{d} \boldsymbol{\xi}^{\prime},
$$

where $d$ is the number of space dimensions of the problem considered.

\subsection{Single-scale models}

Consider now the specialization of (5)-(8) to a single spatial scale (the small scale) so that for any expansion function $\phi^{(i)}$ we have $\nabla_{\boldsymbol{\xi}} \phi^{(i)} \equiv 0$. In this case, $P^{(1)} \equiv P_{1}(t)$, and its gradient disappears from the small scale flow equations in (6). These reduce to the well-known equations for variable density, zero Mach number flow with background compression, i.e.,

$$
\begin{aligned}
\rho_{t}+\nabla_{\boldsymbol{x}} \cdot(\rho \boldsymbol{v}) & =0, \\
(\rho \boldsymbol{v})_{t}+\nabla_{\boldsymbol{x}} \cdot(\rho \boldsymbol{v} \circ \boldsymbol{v})+\nabla_{\boldsymbol{x}} p^{(2)} & =0 \\
\nabla_{\boldsymbol{x}} \cdot \boldsymbol{v} & =-\frac{1}{\gamma P_{0}} \frac{\mathrm{d} P_{0}}{\mathrm{~d} t} .
\end{aligned}
$$

Consider, on the other hand, the specialization of (4) to allow only for long-wave dependencies on $\boldsymbol{\xi}$, requiring $\nabla_{\boldsymbol{x}} \phi^{(i)} \equiv 0$ for all of the expansion functions. Then $\mathrm{d} P_{0} / \mathrm{d} t \equiv 0$, i.e., $P_{0} \equiv P_{\infty}=$ const., and $\rho^{(0)}=\rho_{0}(\boldsymbol{\xi})$ from $(6)_{3}$ and $(6)_{1}$, respectively. The long wave equations from (8) reduce to the equations of linear acoustics with spatially varying speed of sound,

$$
\begin{array}{r}
\rho_{0} \boldsymbol{v}_{t}+\nabla_{\boldsymbol{\xi}} P^{(1)}=0, \\
P_{t}^{(1)}+\gamma P_{\infty} \nabla_{\boldsymbol{\xi}} \cdot \boldsymbol{v}=0 .
\end{array}
$$

In these equations,

$$
P_{\infty}=\text { const., } \quad \rho_{0}=\rho_{0}(\boldsymbol{\xi}),
$$

and with them the sound speed $c(\boldsymbol{\xi})=\sqrt{\gamma P_{\infty} / \rho_{0}(\boldsymbol{\xi})}$, are to be specified together with the initial data and boundary conditions for $\left(P^{(1)}, \boldsymbol{v}\right)$ to define a closed problem.

Thus, by specialization of the general multiple scales ansatz in (4) to two different single-scale versions we have recovered from (5)-(8) two well-known sets of equations from theoretical fluid mechanics. This "mechanism" is the basis for the unified approach to meteorological modelling proposed in [14], and described in more detail in Section 2 below.

\subsection{Scale interactions for variable density flows}

Schochet discusses the state of the art of the rigorous analysis of low Mach number asymptotics elsewhere in this volume [34]. He shows, in particular, that long wave acoustics and small scale quasi-incompressible flow as described by the multiple space scale asymptotics of the last section decouple entirely if homentropic entropy (or sufficiently small entropy variation) is assumed. Clearly, at least for the combustion application discussed 
in Section 1.2 such an assumption is inappropriate: temperature variations due combustion are as large as several hundred degrees, and at almost constant pressure the equation of state enforces similarly large density variations.

When dependencies on both $\boldsymbol{x}$ and $\boldsymbol{\xi}$ are relevant and when there are leading order density variations on the small scale, the long wave acoustic and the small scale quasi-incompressible flow modes do not decouple as we will show in this section (see also [12]).

\section{Baroclinic vorticity generation}

Consider the effect of the acoustic pressure gradient $\nabla_{\xi} P^{(1)}$ in $(6)_{2}$. Obviously, the acoustic pressure gradient changes the momentum of parcels of the gas by the same amount in a given time, independent of whether they have low or high density. The same change of momentum for neighboring light and heavy parcels, however, requires a larger change of velocity for the lighter one. As a consequence, there is vorticity generation wherever the small scale density gradient is not aligned with the acoustic pressure gradient. Of course, this explanation is just an interpretation of the well-known baroclinic source term $\frac{1}{\rho^{2}} \nabla \rho \times \nabla p$ in the vorticity transport equation (see, e.g., [17]). In the present leading order asymptotic representation this term will read $\frac{1}{\left.\rho^{(0)}\right)^{2}} \nabla_{\boldsymbol{x}} \rho^{(0)} \times\left(\nabla_{\boldsymbol{x}} p^{(2)}+\nabla_{\boldsymbol{\xi}} P^{(1)}\right)$. As required by rigorous theory [34], the term vanishes if the density distribution does not feature small scale variation, i.e., when $\nabla_{\boldsymbol{x}} \rho^{(0)} \equiv 0$.

Nonlinear energy flux averages

There is also an influence of the small scale flow onto the longwave acoustic modes. To reveal this effect we observe that the momentum equation in $(8)_{1}$ involves the time derivative of the averaged momentum, $\overline{\rho \boldsymbol{v}}$, whereas the energy flux in (8) 2 involves the averaged velocity, $\overline{\boldsymbol{v}}$. If both, $\rho$ and $\boldsymbol{v}$ are nontrivial functions of the small scale variable, the relation between these quantities reads

$$
\overline{\rho \boldsymbol{v}}=\bar{\rho} \overline{\boldsymbol{v}}+\overline{\widetilde{\rho}} \widetilde{\boldsymbol{v}}
$$

and it depends explicitly on correlation of the small scale density and velocity fluctuations

$$
\widetilde{\rho}=\rho-\bar{\rho} \quad \text { and } \quad \widetilde{\boldsymbol{v}}=\boldsymbol{v}-\overline{\boldsymbol{v}} .
$$

By inserting the decomposition (13) in (8) 2 the long wave modes will be described by the

Long wave acoustics with scale interaction

$$
\begin{aligned}
(\overline{\rho \boldsymbol{v}})_{t}+\nabla_{\boldsymbol{\xi}} P^{(1)} & =0, \\
P_{t}^{(1)}+\nabla_{\boldsymbol{\xi}} \cdot\left(\overline{c^{2}} \overline{\rho \boldsymbol{v}}\right) & =\nabla_{\boldsymbol{\xi}} \cdot\left(\overline{c^{2}} \overline{\widetilde{\rho} \widetilde{\boldsymbol{v}}}\right),
\end{aligned}
$$

where $\overline{c^{2}}=\overline{\gamma P_{\infty} / \rho}$. This completes the derivation of the mutual coupling between long wave acoustic modes and the underlying small scale quasi-incompressible flow in the presence of small scale leading order density variations.

\section{Atmospheric Flows}

In this section we reveal how the general approach of multiple scales asymptotics that has proven useful in low Mach number theory also helps to understand many aspects of meteorological modelling in a mathematically transparent way. Following [13] we will first discuss variants of "anelastic constraints" in Section 2.1, which are the analogue of the zero velocity divergence constraint in incompressible flows. We will then consider in Section 2.2 the fact that, in addition to the Mach number, there are several nondimensional characteristic numbers playing a central role in meteorological modelling. This motivates a brief discussion of the role of distinguished limits in asymptotic analyses of multi-parameter systems. Section 2.3 then explains how a judicious 
choice of distinguished limits for the relevant meteorological parameters and a general multiple scales asymptotic ansatz allow us to recover a wide range of well-known meteorological model equations by specializing this multiple scales ansatz to include only a single horizontal, a single vertical, and a single time scale. Going beyond such "single scale" models we recount briefly some results from [22] on the construction of systematic multiscale models for the tropics in Section 2.4.

\subsection{Divergence constraints}

Apart from the derivation of entire closed sets of simplified model equations for specific atmospheric flow regimes, it is interesting to abstractly discuss the meteorological analogue of the zero velocity divergence constraint which is so ubiquitous in low Mach number models. This is the topic of the present subsection.

A large number of simplified models of theoretical meteorology include the so-called anelastic constraint,

$$
\nabla \cdot(\bar{\rho}(z) \boldsymbol{v})=\bar{\rho}(z) \nabla \cdot \boldsymbol{v}+w \frac{\mathrm{d} \bar{\rho}}{\mathrm{d} z}=0 .
$$

Here $\bar{\rho}(z)$ is the horizontally averaged density stratification, $z$ is the vertical space coordinate. The splitting of the divergence into two contributions, as carried out in the equation above, reveals the physical meaning of the anelastic constraint: as a parcel of air moves vertically over a sufficient distance it experiences a drop of the environmental density and pressure and expands accordingly with a nonzero divergence. Notice that quite generally $\frac{\mathrm{d} \bar{\rho}}{\mathrm{d} z}<0$ for reasons of stability of the stratification.

The anelastic constraint in (16), or variants of it, are found in many simplified models for atmospheric applications, even if these models differ substantially in the way they approximate, e.g., the momentum or energy balances. The original derivation of the anelastic constraint is attributed to [2]. There is a rich amount of literature on the subject aiming at clarification of its limitations and at extending and generalizing the concept, see e.g., $[1,8,19,26]$ and the references therein. To shed some light onto this constraint and its relation to low Mach number theory we follow the arguments given in $[4,13]$.

For simplicity we restrict here to the compressible Euler equations as given in (2), yet we include the influence of gravity and the possibility of distributed energy sources, and we allow for a general equation of state (instead of assuming (3)). Thus, we consider

$$
\begin{aligned}
\rho_{t}+\nabla \cdot(\rho \boldsymbol{v}) & =0, \\
(\rho \boldsymbol{v})_{t}+\nabla \cdot(\rho \boldsymbol{v} \circ \boldsymbol{v})+\frac{1}{\mathrm{M}^{2}}(\nabla p+\rho \nabla \phi) & =0, \\
(\rho e)_{t}+\nabla \cdot([\rho e+p] \boldsymbol{v}) & =S_{\rho e} ;
\end{aligned}
$$

with the equation of state

$$
\rho e=\rho(E(p, \rho)+\phi)+\mathrm{M}^{2} \frac{\rho \boldsymbol{v}^{2}}{2} .
$$

Here $E(p, \rho)$ is the specific internal energy of the fluid, and $\phi(\boldsymbol{x})$ is the gravitational potential. Furthermore we have assumed that the characteristic length for nondimensionalization matches the pressure scale height

$$
h_{\mathrm{sc}}=\frac{p_{\mathrm{ref}}}{\rho_{\mathrm{ref}}|\nabla \phi|_{\mathrm{ref}}} .
$$

This is a typical vertical distance over which the pressure drops appreciably in a (nearly) hydrostatic atmosphere. With this scaling $\sqrt{|\nabla \phi|_{\text {ref }} h_{\mathrm{sc}}}=\sqrt{p_{\text {ref }} / \rho_{\text {ref }}}$ so that the speed of barotropic gravity waves, $c_{g_{\text {ref }}}=\sqrt{|\nabla \phi|_{\text {ref }} h_{\text {sc }}}$, is proportional to a typical sound speed, $c_{\text {ref }}=\sqrt{\gamma p_{\text {ref }} / \rho_{\text {ref }}}$, and the Mach and Froude numbers, $\mathrm{M}=u_{\text {ref }} / c_{\text {ref }}$ and $\mathrm{Fr}=u_{\text {ref }} / c_{g_{\text {ref }}}$, coincide. This has been used explicitly in $(17)_{2},(18)$ in that we have replaced $\frac{1}{\mathrm{Fr}^{2}} \rho \nabla \phi$ and $\frac{\mathrm{M}^{2}}{\mathrm{Fr}^{2}} \rho \phi$ accordingly. (Barotropic gravity waves are characterized by vertically homogeneous horizontal velocities.) 
Consider now the evolution equation for the pressure, $p$, which can be derived from the above equations via straight-forward manipulations.

$$
p_{t}+\boldsymbol{v} \cdot \nabla p+\rho c^{2} \nabla \cdot \boldsymbol{v}=0,
$$

where $c^{2}=\left(p / \rho-\rho E_{\rho}\right) / \rho E_{p}$ is the square of the sound speed, given the general equation of state from (18). Obviously, under the minimal assumption that local pressure variations are small, i.e., $\left|p_{t}\right| \ll|\boldsymbol{v} \cdot \nabla p|,\left|\rho c^{2} \nabla \cdot \boldsymbol{v}\right|$, we have a divergence constraint for the velocity,

$$
\nabla \cdot \boldsymbol{v}+\frac{1}{\rho c^{2}} \boldsymbol{v} \cdot \nabla p=0
$$

As in the zero Mach number limit for "ordinary fluid dynamics" discussed in the previous section, the condition of small pressure variations is much more generally met than the more stringent assumption of small density variations. If there are sizeable fluctuations of entropy or, in meteorological terms, of potential temperature, then we may have large density variations even though the pressure is nearly constant.

Under what conditions will the constraint in (21) take the form of a complete divergence, so that $\nabla \cdot(\alpha \boldsymbol{v})=0$ for some suitable function $\alpha$ ? As $\rho c^{2}$ is a thermodynamic variable, we may consider it a function of $(p, \rho)$, and it is easily shown that

$$
\nabla \cdot(\alpha(p) \boldsymbol{v})=0
$$

provided that there is a function $\kappa(p)$, so that

$$
\frac{1}{\rho c^{2}}=\kappa(p)=\left(\frac{1}{\alpha} \frac{\mathrm{d} \alpha}{\mathrm{d} p}\right)
$$

There are two meteorologically important situations in which such a function $\kappa(p)$ exists at least to leading order in a perturbation analysis. The first is trivial: if we restrict to an ideal gas with constant specific heat capacities, then $\rho c^{2}=\gamma p$, and one has

$$
\alpha(p)=p^{1 / \gamma}, \quad \text { i.e., } \quad \nabla \cdot\left(p^{1 / \gamma} \boldsymbol{v}\right)=0 .
$$

If in addition we restrict to (nearly) homentropic flow, so that $p^{1 / \gamma} / \rho=$ const., except for small perturbations, then (22) is equivalent to the original constraint in (16), with $\bar{\rho}$ the leading order density distribution.

In the second case, the two constraints are, however, not equivalent. Consider to this end the (realistic) situation of a stably stratified atmosphere in which pressure and density are independent functions of height except for small perturbations, i.e.,

$$
\rho=\bar{\rho}(z)+\text { h.o.t., } \quad \text { and } \quad p=\bar{p}(z)+\text { h.o.t. }
$$

Generally, the pressure will monotonously decrease with height, so that the pressure distribution $\bar{p}$ is invertible, and the leading order density may be expressed as a function of the leading order pressure,

$$
\bar{\rho}(z)=: \widehat{\rho}(\bar{p}(z)) .
$$

Now $\left(\rho c^{2}\right)(p, \rho)$, being a thermodynamic function, may to leading order be expressed as a function of $\bar{p}$ alone, i.e.,

$$
\rho c^{2}=\left(\rho c^{2}\right)(\bar{p}, \widehat{\rho}(\bar{p}))=: \widehat{\rho c^{2}}(\bar{p}) .
$$

Then, a function $\alpha(p)$ entering the divergence constraint in (22) will exist, but generally $\alpha(p) \neq \widehat{\rho}(p)$ and the constraints in (16) and (22) will again not be compatible with each other. 
Durran [8], suggests a slightly different formulation of a divergence constraint, called the "pseudo-incompressibility"-constraint. For an ideal gas with constant specific heat capacities, i.e., for the equation of state from (3), the potential temperature, $\theta$, is defined as

$$
\theta=\frac{p^{1 / \gamma}}{\rho},
$$

and it is a direct function of the thermodynamic entropy. The pseudo-incompressible constraint reads

$$
\nabla \cdot(\bar{\rho} \bar{\theta} \boldsymbol{v})=0 .
$$

Obviously, if one defines an averaged potential temperature $\bar{\theta}$ via (28) as

$$
\bar{\theta}=\frac{\bar{p}^{1 / \gamma}}{\bar{\rho}},
$$

with $\bar{p}, \bar{\rho}$ representing horizontal averages, then pseudo-incompressibility is equivalent with $\boldsymbol{v}$ satisfying the divergence constraint (24). Notice, however, that then $\bar{\theta}$ is generally not the horizontal average of $\theta$ !

Anelastic constraints are generally part of simplified meteorological models when the characteristic length scale, $\ell_{\text {ref }}$, considered is acoustically compact, given the considered characteristic time scale, $t_{\text {ref }}$, i.e., when $\ell_{\text {ref }} / t_{\text {ref }} \ll c_{\text {ref }}$.

\subsection{Distinguished limits}

Next we summarize the unified approach to meteorological modelling proposed in [13-15]. We begin with a basic dimensional analysis for a set of globally given characteristic quantities that are independent of the particular length and time scales of any specific atmospheric flow phenomenon.

\subsubsection{Globally valid characteristic quantities}

Characteristic flow velocity magnitudes in the atmosphere outside of upper tropospheric jets are of the order of $10 \mathrm{~m} / \mathrm{s}$, so that, with a typical sound speed around $300 \mathrm{~m} / \mathrm{s}$ a typical flow Mach number is $\mathrm{M} \sim 1 / 30$. The velocity scale of $10 \mathrm{~m} / \mathrm{s}$ is set roughly by the so-called "thermal wind" induced by the global equator-to-pole temperature gradient in a geostrophically balanced flow, (see, e.g., [28]).

The Mach number is, however, just one of several (singular) nondimensional characteristic numbers relevant for atmospheric flows as seen in a quick dimensional analysis [14]. Tables 1 and 2 list sets of characteristic quantities for the rotating earth and for the aerothermodynamics of the dry atmosphere.

Given these universal characteristic scales and observing that they involve as the basic physical dimensions only mass, time, and length, we can form three independent characteristic numbers. A revealing choice of combinations is

$$
\begin{aligned}
& \frac{c_{\mathrm{ref}}}{\Omega a} \sim 0.5 \sim 1, \\
& \frac{u_{\mathrm{ref}}}{c_{\mathrm{ref}}} \sim 3 \times 10^{-2} \ll 1, \\
& \frac{a \Omega^{2}}{g} \sim 6 \times 10^{-3} \ll 1,
\end{aligned}
$$

where

is proportional to the speed of sound.

$$
c_{\text {ref }}=\sqrt{p_{\text {ref }} / \rho_{\text {ref }}}
$$


TABLe 1. Properties of the rotating earth.

\begin{tabular}{|c|c|c|c|}
\hline Earth's radius & $a$ & $\sim$ & $6 \times 10^{6} \mathrm{~m}$ \\
\hline Rotation frequency & $\Omega$ & $\sim$ & $10^{-4} \mathrm{~s}^{-1}$ \\
\hline Acceleration of gravity & $g$ & $\sim$ & $10 \mathrm{~m} / \mathrm{s}^{2}$ \\
\hline
\end{tabular}

TABLE 2. Aerothermodynamic conditions.

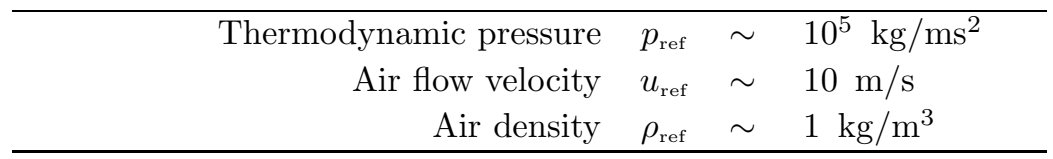

The first parameter is the ratio of the sound speed vs. the circumferential rotation speed of the earth's points on the equator, the second is the Mach number, while the third is the acceleration ratio between the centripetal acceleration due to the earth's rotation near the equator and the acceleration of gravity.

\subsubsection{Distinguished limits vs. multi-parameter expansions}

Planning to approach the modelling of atmospheric flows using low Mach number expansions, we should account for the fact that the acceleration ratio is a second small parameter of the system. One option would be to attempt a two parameter expansion, which allows both parameters to vary independently. Generally, if a system is characterized by two small parameters, $\varepsilon, \delta \ll 1$, say, then such an expansion reads

$$
\mathbf{U}(\boldsymbol{x}, t ; \varepsilon, \delta)=\mathbf{U}^{(0)}(\boldsymbol{x}, t)+\varepsilon \mathbf{U}^{(1,0)}(\boldsymbol{x}, t)+\delta \mathbf{U}^{(0,1)}(\boldsymbol{x}, t)+\ldots
$$

where $\mathbf{U}$ represents the set of unknowns. As argued in $[14,15]$ such an independent two parameter expansion requires that a unique limit $\mathbf{U}^{(0)}(\cdot)=\lim _{\varepsilon, \delta \rightarrow 0} \mathbf{U}(\cdot ; \varepsilon, \delta)$ exists, and that $\mathbf{U}$ is $n$ times differentiable w.r.t. $(\varepsilon, \delta)$ at $\varepsilon=\delta=0$ for a meaningful expansion up to $n$th order in both parameters.

Unfortunately, even for the extremely simple example of the linear oscillator with small mass and small damping, described by

$$
\varepsilon \ddot{y}+\delta \dot{y}+y=f(t)
$$

a unique leading order limit solution $y^{(0)}$ does not exist. This is easily seen by letting (i) first $\varepsilon$, then $\delta$ go to zero and (ii) choosing the opposite sequence of limits. In the former case one will obtain a strongly damped, non-oscillatory motion with exponential decay with a characteristic decay time proportional to $\delta$. If the damping parameter, $\delta$, vanishes first, we obtain an oscillatory undamped solution with a characteristic oscillation time scale proportional to $\sqrt{\varepsilon}$.

A more general approach, requiring less stringent assumptions regarding the solutions' dependence on the small parameters, employs distinguished limits, so that $\delta=\hat{\delta}(\varepsilon)$ as $\varepsilon \rightarrow 0$. The existence of an $\varepsilon$-expansion in this case merely requires differentiability of the solution w.r.t. $\varepsilon$ along the path $\delta=\hat{\delta}(\varepsilon)$, i.e., the existence of the associated generalized directional derivatives. And these, of course, exist under much weaker conditions than complete gradients.

Along paths given by different distinguished limits one may expect a different asymptotic limiting behavior, and the challenge in practice is to find those distinguished limits that capture the physically relevant phenomena for the applications considered. In this context see also the remarks on "richt limits" in [34]. 


\subsection{Meteorological modelling via multiple scales asymptotics}

\subsubsection{Distinguished limit and multiple scales ansatz}

In [14] the author proposes a particular distinguished limit for the parameters in (31) which, in combination with systematic multiple scales expansions, has turned out to capture a considerable variety of the scaling regimes studied in the classical and modern meteorological literature. In fact, we let

$$
\mathrm{M}=\frac{u_{\mathrm{ref}}}{c_{\mathrm{ref}}}=O\left(\varepsilon^{2}\right), \quad \frac{a \Omega^{2}}{g}=O\left(\varepsilon^{3}\right) \quad \text { as } \quad \varepsilon \rightarrow 0 .
$$

In practice, $\varepsilon \sim 1 / 8 \ldots 1 / 5$.

With these distinguished limits, and using the aerothermodynamic reference values from Table 2 to nondimensionalize pressure, density, and velocity, the pressure scale height, $h_{\mathrm{sc}}$, from (19) to scale the space coordinate, and $t_{\text {ref }}=h_{\mathrm{sc}} / u_{\mathrm{ref}}$ as the unit of time, the governing equations for three-dimensional compressible flow under the influence of gravity and rotation read

$$
\begin{aligned}
\boldsymbol{v}_{t}+\boldsymbol{v} \cdot \nabla \boldsymbol{v}+\varepsilon \boldsymbol{\Omega} \times \boldsymbol{v}+\frac{1}{\varepsilon^{4}} \frac{1}{\rho} \nabla p & =\boldsymbol{S}_{\boldsymbol{v}}-\frac{1}{\varepsilon^{4}} \boldsymbol{k} \\
p_{t}+\boldsymbol{v} \cdot \nabla p+\gamma p \nabla \cdot \boldsymbol{v} & =S_{p} \\
\theta_{t}+\boldsymbol{v} \cdot \nabla \theta & =S_{\theta}
\end{aligned}
$$

with the equation of state for density,

$$
\rho=\frac{p^{1 / \gamma}}{\theta}
$$

Here we have subsumed all effects of molecular and turbulent transport, radiation, latent heat release from condensation, etc., in explicit source terms, $\boldsymbol{S}_{\boldsymbol{v}}, S_{p}, S_{\theta}$, the detailed structure of which we will not discuss further in this paper. Of course, in practice these terms will give rise to additional asymptotic scalings, see e.g., $[18,19]$. Related analyses using the present approach are work in progress.

With (36), (37), we have a set of equations characterized by a single small parameter, $\varepsilon$. The limit $\varepsilon \rightarrow 0$ is singular as is evident from the degeneration of the momentum equation $(36)_{1}$ in this limit. On the other hand, we know that this same set of equations describes atmospheric flow phenomena associated with very diverse length and time scales as illustrated in Figure 5. Given the positive experience with the low Mach number limit as described in Section 1 a general multiple scales asymptotic ansatz was proposed in [14] in an attempt to capture the multitude of length and time scales of atmospheric flows,

$$
\mathbf{U}(t, \boldsymbol{x}, z ; \varepsilon)=\sum_{i=0}^{n} \phi^{(i)}(\varepsilon) \mathbf{U}^{(i)}\left(\frac{t}{\varepsilon}, t, \varepsilon t, \varepsilon^{2} t, \ldots, \frac{\boldsymbol{x}}{\varepsilon}, \boldsymbol{x}, \varepsilon \boldsymbol{x}, \varepsilon^{2} \boldsymbol{x}, \ldots, \frac{z}{\varepsilon}, z\right)+o\left(\phi^{(n+1)}(\varepsilon)\right) .
$$

Here the $\phi^{(i)}$ form an asymptotic sequence, so that

$$
\phi^{(i+1)}(\varepsilon)=o\left(\phi^{(i)}(\varepsilon)\right) \quad \text { as } \quad \varepsilon \rightarrow 0 .
$$

Thus far, simple powers of $\varepsilon$, as in $\phi^{(i)}(\varepsilon)=\varepsilon^{i}$ or $\phi^{(i)}(\varepsilon)=\varepsilon^{i+\frac{1}{2}}$ have proven sufficient in applications of the present general ansatz in $[14,15]$, while the analysis of near-equatorial flows in [22] requires fractional powers of $\varepsilon$ also in the coordinate scalings (see also Sect. 2.4 below). This does not preclude the necessity to employ more complex scaling functions in other cases, such as boundary layer theory $[11,25]$. 


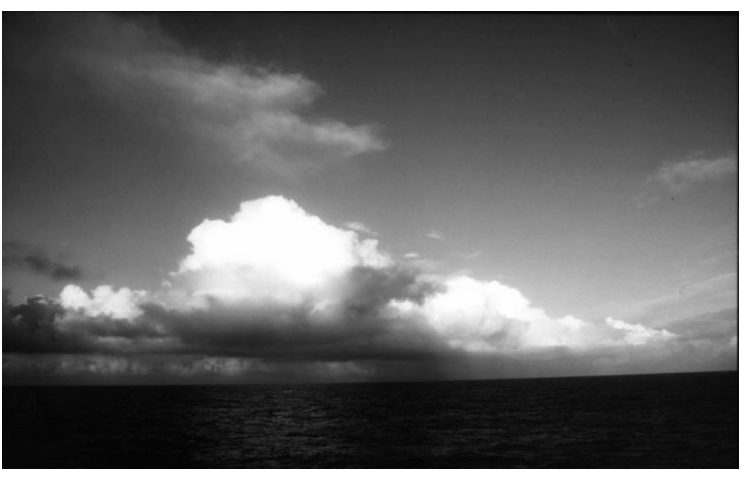

$10 \mathrm{~km} / 20 \mathrm{~min}$

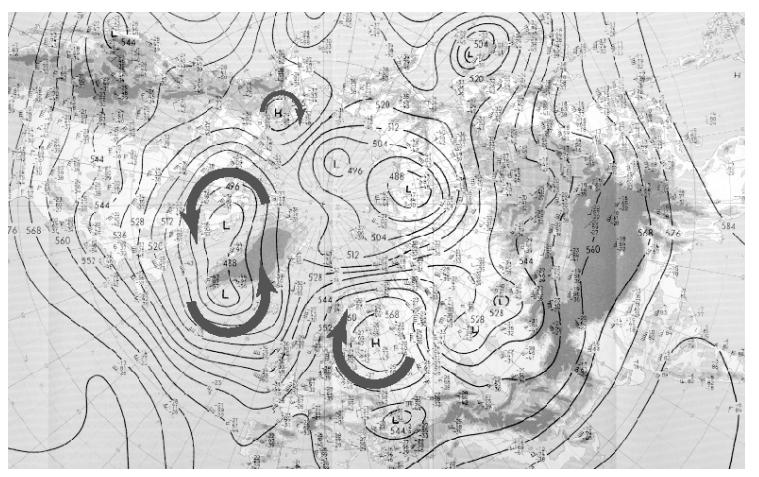

$1,000 \mathrm{~km} / 2$ days

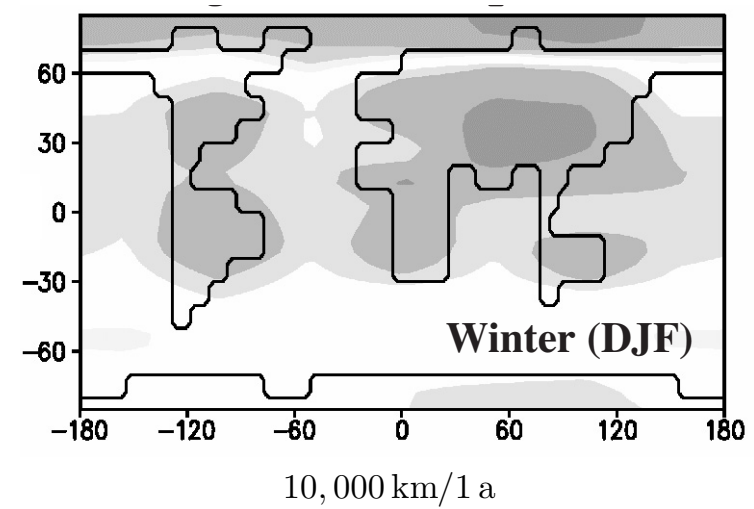

Figure 5. Scale separated phenomena in the atmosphere: Photograph of a cloud formation process on scales of $10 \mathrm{~km}$ and $20 \mathrm{~min}$ (top left), courtesy of P.T. Taylor, J. Rennell Division, Southampton Oceanography Centre, UK. Result of a "synoptic analysis" of cyclonic and anti-cyclonic motions on scales of $1000 \mathrm{~km}$ and 2 days (top right). Shown are contours of the geopotential hight of the 500 mbar surface of constant pressure, courtesy of P. Névir, Dept. of Meteorology, Freie Universität Berlin, Germany. Change of the december-january-february mean temperature over several thousand years taken from a long-term climate simulation using the CLIMBER-2 model [29], courtesy of A. Ganopolski, S. Rahmstorf, Potsdam Institute for Climate Impact Research, Germany.

\subsubsection{Validation of the approach against well-established models of theoretical meteorology}

The approach chosen here is to be validated before we embark on ambitious multiscale analyses. There is a large number of simplified models of theoretical meteorology that address selected phenomena that are associated with specific horizontal, vertical, and time scales. When specialized, in this sense, to a single scale ansatz - that is, specialized to cover a single horizontal, a single vertical, and a single time scale - our ansatz from (38) should allow us to recover many of these well-established simplified models. In fact, a number of relevant examples have been worked out in [13-16,22], and we recount these results briefly here, without providing details of the derivations.

Mesoscale anelastic and buoyancy-controlled models

Processes of cloud formation (see Fig. 5, top left) develop on characteristic scales of $100 \mathrm{~m}$ to $10 \mathrm{~km}$, both horizontally and vertically, and on time scales of up to $20 \mathrm{~min}$. These are also the scales used in nondimensionalizing the governing equations in (36). Therefore, the special case of our solution ansatz in (38) with expansion 
functions

$$
\mathbf{U}^{(i)}(\boldsymbol{x}, z, t)
$$

should reproduce the model equations generally considered applicable to such mesoscale flows. In fact, two different sets of governing equations are obtained, depending on additional scaling assumptions regarding the stability of the atmospheric stratification. The stratification is neutral in the case of a homentropic fluid. Fluid elements may then freely move upwards or downwards, never experiencing any (positive or negative) net buoyancy force. The stratification is stable if the entropy (potential temperature) increases with height. In this case, a parcel lifted upwards will, after its pressure has adjusted to the new environment, be colder and heavier than its surroundings, and it will therefore experience a net downward restoring force.

For nearly neutral stratification, specifically when

$$
\theta=1+\varepsilon^{4} \theta^{(4)}+o\left(\varepsilon^{4}\right)
$$

the asymptotic analysis yields, at leading order, the "classical" anelastic model equations [26], i.e.,

$$
\begin{gathered}
\boldsymbol{u}_{t}+\boldsymbol{u} \cdot \nabla_{\|} \boldsymbol{u}+w \boldsymbol{u}_{z}+\nabla_{\|} \pi=S_{u}, \\
w_{t}+\boldsymbol{u} \cdot \nabla_{\|} w+w w_{z}+\pi_{z}=-\theta^{\prime}+S_{w}, \\
\theta_{t}^{\prime}+\boldsymbol{u} \cdot \nabla_{\|} \theta^{\prime}+w \theta_{z}^{\prime}=S_{\theta}^{\prime}, \\
\nabla_{\|} \cdot\left(\rho_{0} \boldsymbol{u}\right)+\left(\rho_{0} w\right)_{z}=0 .
\end{gathered}
$$

Here

and

$$
\theta^{\prime} \equiv \theta^{(4)}, \quad \pi \equiv p^{(4)} / \rho_{0}, \quad \rho^{(0)}(\boldsymbol{x}, z, t) \equiv \rho_{0}(z)=\exp (-\gamma z)
$$

$$
\boldsymbol{u}=(\mathbf{1}-\boldsymbol{k} \circ \boldsymbol{k}) \boldsymbol{v}, \quad w=\boldsymbol{k} \cdot \boldsymbol{v}, \quad \nabla_{\|}=\nabla(\mathbf{1}-\boldsymbol{k} \circ \boldsymbol{k}),
$$

are the horizontal components of the flow velocity and the gradient operator, with $\boldsymbol{k}$ the vertical unit vector.

We observe that $(42)_{4}$ is a version of the anelastic constraints discussed in Section 2.1, and that these model equations feature a fully threedimensional, unsteady momentum balance. In terms of its mathematical structure, this is possibly the meteorological model closest to the classical incompressible flow equations.

If the stratification is stronger, and also more realistic, a different leading order set of equations obtains. In fact, if we allow for entropy variations large compared to $\varepsilon^{4}$, then one finds first that these larger variations have to be stratified purely vertically,

$$
\theta=1+\varepsilon^{\alpha} \Theta(z)+o\left(\varepsilon^{\alpha}\right)
$$

with $0 \leq \alpha<4$, and that the vertical momentum equation degenerates to yield $[6,10,40]$,

$$
\begin{aligned}
\boldsymbol{u}_{t}+\boldsymbol{u} \cdot \nabla \boldsymbol{u}+w \boldsymbol{u}_{z}+\nabla \pi & =\boldsymbol{S}_{u}, \\
w \frac{\mathrm{d} \Theta}{\mathrm{d} z} & =S_{\theta}^{(\alpha)}, \\
\nabla \cdot\left(\rho_{0} \boldsymbol{u}\right)+\left(\rho_{0} w\right)_{z} & =0 .
\end{aligned}
$$

These equations describe vertically sheared, essentially horizontal motions, with vertical motion induced only by thermal heat sources represented by $S_{\theta}^{(\alpha)}$. The interpretation of (46) is that a parcel of air that receives or looses heat changes its entropy and then moves vertically to its new level of neutral buoyancy. With vertical stratifications of $\theta$ stronger than $O\left(\varepsilon^{4}\right)$ this process occurs on time scales much shorter than the 20 min considered here, so that the adjustment of the vertical velocity to external heat sources is immediate. Notice that vertical motion is inhibited for adiabatic flows, $S_{\theta} \equiv 0$. The anelastic constraint holds in this regime, too, according 
to $(46)_{3}$. Many simplified models for near-equatorial flows are based on this kind of approximation, which goes under the term "weak temperature gradient approximation" in $[5,36]$.

\section{Quasi-geostrophic theory}

This classical theory aims at describing the evolution of high- and low pressure systems in the middle latitudes, which are known to be the main determinants for the observed daily weather patterns. With the coordinate scalings

$$
\mathbf{U}^{(i)}\left(\varepsilon^{2} t, \varepsilon^{2} \boldsymbol{x}, z\right)=\mathbf{U}^{(i)}(\tau, \boldsymbol{\xi}, z),
$$

and the following additional assumption regarding the stability of the atmospheric stratification

$$
\theta(\boldsymbol{x}, z, t ; \varepsilon)=1+\varepsilon^{2} \Theta_{2}(z)+\varepsilon^{3} \theta^{(3)}(\tau, \boldsymbol{\xi}, z)+o\left(\varepsilon^{3}\right),
$$

we recover the derivations in [28], which are based on a low Rossby number expansion. The characteristic length and time scales represented by the stretched coordinates, $\boldsymbol{\xi}, \tau$, are of the order of $1000 \mathrm{~km}$ and $48 \mathrm{~h}$. In particular, the characteristic length is still much smaller than the global scale $(10000-40000 \mathrm{~km})$. As a consequence, the resulting theory is formally applicable only to flows in limited regions, which are well approximated by considering the so-called tangent plane approximation. This approximation, which is consistently obtained in the asymptotic expansions, considers planar flows and accounts for the curvature of the earth's surface only through the meridional (north-south) variation of the Coriolis parameter.

The result of a formal asymptotic analysis based on (47) is a transport equation:

$$
\left(\partial_{\tau}+\boldsymbol{u} \cdot \nabla_{\boldsymbol{\xi}}\right) q=0
$$

for the potential vorticity

$$
q=\zeta+\beta \eta+\frac{1}{\rho_{0}} \frac{\partial}{\partial z}\left(\frac{f_{0} \rho_{0}}{\mathrm{~d} \Theta_{2} / \mathrm{d} z} \theta^{(3)}\right) .
$$

Here $\boldsymbol{u}$ is the leading order horizontal flow velocity, which satisfies the geostrophic balance between the Coriolis force and the horizontal pressure gradient, so that

$$
\boldsymbol{u}=f_{0} \boldsymbol{k} \times \nabla_{\boldsymbol{\xi}} \pi^{(3)} .
$$

In these equations $\zeta=\boldsymbol{k} \cdot \nabla_{\boldsymbol{\xi}} \times \boldsymbol{u}$ is its vertical vorticity component, $\Theta_{2}, \theta^{(3)}$ are the second and third order perturbations of the potential temperature, $\rho_{0}(z)$ is the leading order background density stratification, $f_{0}$ is the leading order Coriolis parameter for the flow in a tangent plane to the earth (i.e., essentially the vertical component of the earth rotation vector), $\eta$ is the meridional (north-south) component of $\boldsymbol{\xi}, \beta$ is the derivative of the Coriolis parameter with respect to $\eta$, and $\pi^{(3)}=p^{(3)} / \rho_{0}$ is a density-scaled third order perturbation pressure. Between $\pi^{(3)}$ and $\theta^{(3)}$ there is the constraint of hydrostatic balance, so that

$$
\frac{\partial \pi^{(3)}}{\partial z}=\theta^{(3)}
$$

A closed system of equations for $q$ and $\pi^{(3)}$ is obtained by eliminating $\theta^{(3)}$ and $\zeta$ from (50) using (52) and

$$
\zeta=f_{0} \nabla_{\boldsymbol{\xi}}^{2} \pi^{(3)} .
$$

This provides an elliptic equation for $\pi^{(3)}$, given the spatial distribution of $q$, viz.,

$$
\nabla_{\xi}^{2} \pi^{(3)}+\frac{1}{\rho_{0}} \frac{\partial}{\partial z}\left(\frac{f_{0} \rho_{0}}{\mathrm{~d} \Theta_{2} / \mathrm{d} z} \frac{\partial \pi^{(3)}}{\partial z}\right)=q-\beta \eta .
$$


Boundary conditions for $\pi^{(3)}$ that would allow one to solve this elliptic equation are discussed extensively, e.g., in [28]. Discussing these in detail is beyond the scope of the present paper.

\section{The Hydrostatic Primivite Equations}

An interesting set of simplified model equations from the point of view of low Mach number theory are the Hydrostatic Primitive Equations (HPE). These equations, to be summarized shortly, form the basis of the majority of current weather prediction codes and global as well as regional climate models. Interestingly, these equations are thus far the only ones that cannot be derived using the multiple scales asymptotics ansatz as summarized in 2.3.1 above.

In fact, the scaling assumptions leading to the HPEs do include a low Mach number assumption, however, only for the vertical velocity. The horizontal velocities are assumed to be characterized by "horizontal flow Mach numbers" of order $O(1)$. The second assumption needed in a derivation of the HPEs is that of a shallow layer of fluid, so that the horizontal and vertical characteristic lengths differ by an asymptotically small scaling factor. Thirdly, the horizontal characteristic length is chosen in the sense of a "rich limit" (see [34], so as to retain the Coriolis effect as an order $O(1)$ contribution in the horizontal momentum equation in the limit.

These scaling considerations can be translated into an asymptotic ansatz using the present distinguished limits from (35), together with the following specialization and modification of the asymptotic solution ansatz from (38). All expansion functions, $U^{(i)}$, have a signature

$$
U^{(i)}(\vartheta, \boldsymbol{X}, z), \quad \text { where } \quad \vartheta=\varepsilon t, \quad \boldsymbol{X}=\varepsilon^{3} \boldsymbol{x},
$$

and pressure, potential temperature, and the vertical and horizontal velocities are expanded as

$$
\left.\begin{array}{rl}
p(t, \boldsymbol{x}, z ; \varepsilon) & =p^{(0)}(\vartheta, \boldsymbol{X}, z)+o(1) \\
\theta(t, \boldsymbol{x}, z ; \varepsilon) & =\theta^{(0)}(\vartheta, \boldsymbol{X}, z)+o(1) \\
\boldsymbol{u}(t, \boldsymbol{x}, z ; \varepsilon) & =\varepsilon^{-2} \boldsymbol{u}^{(-2)}(\vartheta, \boldsymbol{X}, z)+o\left(\varepsilon^{-2}\right) \\
w(t, \boldsymbol{x}, z ; \varepsilon) & =\varepsilon w^{(1)}(\vartheta, \boldsymbol{X}, z)+o(\varepsilon)
\end{array}\right\} \quad \text { as } \quad \varepsilon \rightarrow 0 .
$$

It is then quite straight-forward to derive the following leading order equations from (36) (we drop the $(\cdot)^{(i)}$ superscripts for convenience of notation),

$$
\begin{array}{r}
\boldsymbol{u}_{\vartheta}+\boldsymbol{u} \cdot \nabla_{\boldsymbol{X}} \boldsymbol{u}+w \boldsymbol{u}_{z}+f(\boldsymbol{X}) \boldsymbol{k} \times \boldsymbol{u}+\frac{1}{\rho} \nabla_{\boldsymbol{X}} p=\boldsymbol{S}_{\boldsymbol{v}}, \\
p_{z}=-\rho, \\
p_{\vartheta}+\boldsymbol{u} \cdot \nabla_{\boldsymbol{X}} p+w p_{z}+\gamma p\left(\nabla_{\boldsymbol{X}} \cdot \boldsymbol{u}+w_{z}\right)=S_{p}, \\
\theta_{\vartheta}+\boldsymbol{u} \cdot \nabla_{\boldsymbol{X}} \theta+w \theta_{z}=S_{\theta} .
\end{array}
$$

Here $f(\boldsymbol{X})$ is the spatially varying Coriolis parameter, and the density is determined by the equation of state

$$
\rho=\frac{p^{1 / \gamma}}{\theta}
$$

These equations provide a simplification of the vertical momentum balance, which reduces to the hydrostatic equation $(57)_{2}$, while they retain the full nonlinear horizontal momentum transport, the Coriolis effect, and effect of long wavelength gravity sound waves (so-called "Lamb waves") as we will see shortly. Notice that 
we have written these equations for a shallow fluid in a flat geometry. The reader may consult [38] for the formulation of the HPEs on the sphere.

The possibility of "acoustic" modes in (57), (58) is seen easily by considering the special case of an atmosphere at rest with homogeneous leading order temperature and neglecting the Coriolis-Term. Thus we let pressure and density in the unperturbed state, $p_{0}, \rho_{0}$ satisfy

$$
T_{0}=\frac{p_{0}}{\rho_{0}} \equiv \text { const. }=1 .
$$

Then

$$
p_{0}(z)=\rho_{0}(z)=\exp (-z), \quad \theta_{0}(z)=\exp \left(\frac{\gamma-1}{\gamma} z\right) .
$$

Now we seek infinitesimal perturbations, $p^{\prime}, \rho^{\prime}, \theta^{\prime}, \boldsymbol{u}^{\prime}, w^{\prime}$, so that

$$
\boldsymbol{u}=\boldsymbol{u}^{\prime}, \quad w=w^{\prime}, \quad p=p_{0}(z)+p^{\prime}, \quad \rho=\rho_{0}(z)+\rho^{\prime}, \quad \theta=\theta_{0}(z)+\theta^{\prime} .
$$

The perturbations will then obey the linearized equations

$$
\begin{aligned}
\boldsymbol{u}_{\vartheta}^{\prime}+\frac{1}{\rho_{0}} \nabla_{\boldsymbol{X}} p^{\prime} & =0, \\
p_{\vartheta}^{\prime}+\gamma p_{0} \nabla_{\boldsymbol{X}} \cdot \boldsymbol{u}^{\prime} & =0, \\
p_{z}^{\prime} & =-\rho^{\prime}, \\
\theta_{\vartheta}^{\prime} & =0,
\end{aligned}
$$

with

$$
\rho^{\prime}=\rho_{0}\left(\frac{1}{\gamma} \frac{p^{\prime}}{p_{0}}-\frac{\theta^{\prime}}{\theta_{0}}\right)
$$

Sound modes are found through the separation of variables ansatz

$$
\theta^{\prime}(\vartheta, \boldsymbol{X}, z) \equiv 0, \quad \rho^{\prime}(\vartheta, \boldsymbol{X}, z)=p^{\prime} / \gamma, \quad p^{\prime}(\vartheta, \boldsymbol{X}, z)=\varphi(z) \widehat{p}(\vartheta, \boldsymbol{X}), \quad \boldsymbol{u}^{\prime}(\vartheta, \boldsymbol{X}, z)=\varphi(z) \widehat{\boldsymbol{u}}(\vartheta, \boldsymbol{X})
$$

With $\phi(z)=\exp (-z / \gamma)$ we satisfy the hydrostatic balance equation $(62)_{3},(63)$, and then the horizontal modes $\widehat{p}, \widehat{\boldsymbol{u}}$ satisfy the linearized acoustic equations for horizontally propagating sound waves.

The hydrostatic primitive equations are also often preferred in practice over anelastic models for two reasons. The first is quite pragmatic: there is a finite speed of signal propagation in the HPEs, so that they can be solved by explicit numerical methods - albeit with operator splitting-type "subcycling" of the fast modes described by the linearized equations from (62). A reason to prefer the HPEs over the quasi-geostrophic model from (49)-(54), is that - in their formulation for the flow of a shallow fluid layer on the sphere - they are uniformly valid across the equator, where the Coriolis term vanishes. The quasi-geostrophic model relies on the geostrophic balance (51), and degenerates as the equator is approached. Notice, however, that there is a wealth of literature on the non-trivial question of the well-posedness of the HPEs, beginning with Oliger and Sundström [27].

\subsection{A multiscale model for the tropics}

The general asymptotic ansatz from (38), of course, is meant to form the basis for the derivation of true multiple scales models, in contrast to the examples from the last section, which considered a single scale for the horizontal and vertical space directions and for time, respectively. The first successful exploration of the approach has led to a group of multiple scales models for near-equatorial flows in [22]. 


\section{The IPESD model}

We recount here the "Intraseasonal Planetary Equatorial Synoptic Dynamics" model (IPESD), which is based on the following single time, multiple length scale ansatz. The expansion functions have the signature

$$
U^{(i)}\left(T_{\mathrm{S}}, \boldsymbol{X}_{\mathrm{S}}, X_{\mathrm{P}}, z\right), \quad \text { where } \quad T_{\mathrm{S}}=\varepsilon^{5 / 2} t, \quad \boldsymbol{X}_{\mathrm{S}}=\varepsilon^{5 / 2} \boldsymbol{x}, \quad X_{\mathrm{P}}=\varepsilon^{7 / 2} x .
$$

The scaling for the smaller scale, represented by $\boldsymbol{X}_{\mathrm{S}}$, is chosen again in the sense of a rich limit, namely such that quasi-geostrophic balance is obtained. As the Coriolis parameter vanishes towards the equator, this requires a systematically larger horizontal scale than it does for the mid-latitude quasi-geostrophic theory, see (47) and the related discussion above.

The expansions of the dependent variables reflect the occurrence of a fractional power of $\varepsilon$ in the coordinate scalings, and we have

$$
\left.\begin{array}{rl}
p(t, \boldsymbol{x}, z ; \varepsilon) & =p_{0}(z)+\varepsilon^{3} p^{(3)}+\varepsilon^{4} p^{(4)}+o\left(\varepsilon^{4}\right) \\
\theta(t, \boldsymbol{x}, z ; \varepsilon) & =1+\varepsilon^{2} \Theta_{2}(z)+\varepsilon^{3} \theta^{(3)}+o\left(\varepsilon^{3}\right) \\
\boldsymbol{u}(t, \boldsymbol{x}, z ; \varepsilon) & =\boldsymbol{u}^{(0)}+\varepsilon \boldsymbol{u}^{(1)}+o(\varepsilon) \\
w(t, \boldsymbol{x}, z ; \varepsilon) & =\varepsilon^{5 / 2} w^{(5 / 2)}+\varepsilon^{7 / 2} w^{(7 / 2)}+o\left(\varepsilon^{7 / 2}\right)
\end{array}\right\} \quad \text { as } \quad \varepsilon \rightarrow 0
$$

with signatures of the expansion functions as in (65) unless specified otherwise. Following [22] we simplify the notation in the sequel according to

$$
\begin{aligned}
\left(\theta^{(3)}, \boldsymbol{u}^{(0)}, \boldsymbol{u}^{(1)}, w^{(5 / 2)}, w^{(7 / 2)}, p^{(3)} / \rho_{0}, p^{(4)} / \rho_{0}\right) & \rightarrow\left(\theta, \boldsymbol{u}, \boldsymbol{u}^{\prime}, w, w^{\prime}, \pi, \pi^{\prime}\right), \\
T_{\mathrm{S}} & \rightarrow t \\
\left(\boldsymbol{X}_{\mathrm{S}}, X_{\mathrm{P}}\right) & \rightarrow(x, y, X), \\
\nabla_{\boldsymbol{X}_{\mathrm{S}}} & \rightarrow \nabla .
\end{aligned}
$$

Then, the "small scales", that is scales of the order of $\sim 1500 \mathrm{~km}$ in this setting, satisfy a geostrophically balanced "Gill-modell" [9,24,37],

$$
\begin{aligned}
\partial_{z} \pi & =\theta, \\
w \frac{\mathrm{d} \Theta_{2}}{\mathrm{~d} z} & =S_{\theta}, \\
-\beta y v+\partial_{x} \pi & =0, \\
\beta y u+\partial_{y} \pi & =0, \\
\partial_{x} u+\partial_{y} v+\frac{1}{\rho_{0}} \partial_{z}\left(\rho_{0} w\right) & =0 .
\end{aligned}
$$

Here $S_{\theta}$ represents effective heat sources, e.g., due to latent heat release or radiation, and $\beta$ is again the meriodonal derivative of the Coriolis parameter (scaled appropriately). We have neglected additional source terms in the two momentum equations $(68)_{3,4}$, here. These were included in the original derivations in [22], but were found not to be important in a detailed study of solution structures related to the so-called "MaddenJulian-Oscillation" in $[3,21]$. Thus we assume the momentum sources to appear only at the next order in the expansions (see below). 
Given the possibly time dependent heat source $S_{\theta}$, satisfying the constraint of vanishing $x$-average,

$$
\overline{S_{\theta}} \equiv 0
$$

the equations in (68) have a straight-forward exact solution for $(\pi, \theta, u, v, w)$ that is unique up to solutions of the homogeneous system (for which $S_{\theta} \equiv 0$ ). It is easily verified that such homogeneous solutions have the following structure,

$$
v=w \equiv 0, \quad(\pi, \theta, u)=(P, \Theta, U)(t, y, z)
$$

where $\Theta, U, P$ are arbitrary functions except for the geostrophic and hydrostatic constraints

$$
\partial_{y} P=-\beta y U, \quad \partial_{z} P=\Theta
$$

Because of the linearity of the equations w.r.t. the unknowns, $(\pi, \theta, u, v, w)$, arbitrary homogeneous solutions may be added to any given solution to obtain another one. Now, given a source distribution $S_{\theta}(x, y, z)$, let

$$
(\tilde{\pi}, \tilde{\theta}, \tilde{u}, \tilde{v}, \tilde{w})\left[S_{\theta}\right](x, y, z) \equiv \text { solutions to }(68) \text { with vanishing } x \text {-averages of }(\pi, \theta, u) \text {. }
$$

In the present multiple scales setting, the source distribution will be parameterized by time, $t$, and by the large scale location, $X$, i.e., it has the signature $S_{\theta}(x, y, z ; t, X)$. Thus the small scale particular solutions from (72) will also have $(t, X)$ as additional parameters.

In practical applications the zonal averages of $(\pi, \theta, u)$ will not vanish identically, and the multiple scales ansatz with zonal coordinates $x$ and $X=\varepsilon x$ allows us to derive evolution equations for these large scale averages. In fact, the two scale solutions are superpositions of the unique particular solutions in (72) with long wave components, so that

$$
(\pi, \theta, u, v, w)(t, x, X, y, z)=(P, \Theta, U, 0,0)(t, X, y, z)+(\tilde{\pi}, \tilde{\theta}, \tilde{u}, \tilde{v}, \tilde{w})\left[S_{\theta}(\cdot ; t, X)\right](x, y, z) .
$$

A closed system of evolution equations for the long wave components is found by imposing sublinear growth conditions on the next order solutions, see [22]. The result is the following system of equatorial long wave equations which include source terms due to nonlinear averages of the zonal momentum and potential temperature advection,

$$
\begin{aligned}
\partial_{t} U+\partial_{X} P-\beta y \overline{v^{\prime}} & =\overline{S_{u}^{\prime}}-\overline{\tilde{D}_{t} \tilde{u}}, \\
\partial_{t} \Theta+\overline{w^{\prime}} \frac{\mathrm{d} \Theta_{2}}{\mathrm{~d} z} & =\overline{S_{\theta}^{\prime}}-\overline{\tilde{D}_{t} \tilde{\theta}}, \\
\beta y U+\partial_{y} P & =0, \\
\partial_{z} P & =\Theta, \\
\partial_{X} U+\partial_{y} \overline{v^{\prime}}+\frac{1}{\rho_{0}} \partial_{z}\left(\rho_{0} \overline{w^{\prime}}\right) & =0 .
\end{aligned}
$$

Here $\overline{v^{\prime}}, \overline{w^{\prime}}$ are the $x$-averages of the next order perturbations of the meridional and vertical velocities (cf. (67)), $\overline{S_{u}^{\prime}}, \overline{S_{\theta}^{\prime}}$ are the large scale effective drag and the averaged next order heat source, and

$$
\tilde{D}_{t}=\tilde{u} \partial_{x}+\tilde{v} \partial_{y}+\tilde{w} \partial_{z}
$$

represents the operator describing nonlinear small scale advection. 
The multiple scales system $(68)-(75)$ describes how synoptic scale $(\sim 1500 \mathrm{~km})$ distributions of effective heat sources, e.g., from radiation and latent heat release in cloud clusters, drive a geostrophically balanced flow. If there is a planetary scale distribution of such systems along the equator, they induce a mean nonlinear advection of zonal momentum and entropy (potential temperature). Together with large scale momentum drag and higher order mean heat sources, these drive planetary equatorial waves. In turn, of course, the vertically sheared zonal mean flow will influence the local heat source distribution, $S_{\theta}(\cdot ; t, X)$, and thus modify the small scale flow.

Biello and Majda [3,21], demonstrate how such multi-scale interactions can trigger long-wave phenomena that do not resemble any of the classical long wave solutions represented by the homogeneous version of (74), but that have striking similarity with the thus far unexplained Madden-Julian-Oscillation [20].

\section{Conclusions}

Motivated by numerous applications, ranging from thermoacoustics, via accelerating flames and the deflagration-to-detonation transition to synoptic-planetary scale interactions in the tropical atmosphere, we have summarized here how formal multiple scales asymptotics supports the mathematical modelling of these phenomena. The emphasis being on low Mach number analyses, we found that in engineering applications the scale separation needed to justify a multiple scales analysis is associated with acoustic and advective processes, respectively. A multiple space, single time scale low Mach number expansion of the compressible flow equations revealed that there are nontrivial interactions of small scale advective motions and long wave acoustics, provided there are leading order density fluctuations on the small scales. Specializations of the multiscale theory to include only the small or only the large scales led naturally to two well-established low Mach number models, namely the variable density incompressible flow equations, and the linear acoustic equations with variable speed of sound, respectively.

For atmospheric flows the situation is much more complex, as there are a number of additional singular small parameters besides the Mach number, all giving rise to asymptotic scale separations. To procede, we have introduced a particular distinguished limit of these small parameters leaving us with a single small parameter $\varepsilon \sim \sqrt{\mathrm{M}}$, which then served as the expansion parameter in the subsequent multiple scales asymptotic analyses. In analogy with the (almost trivial) recovery of the incompressible and acoustic equations in the "pure" low Mach number setting, we have demonstrated how various specializations of a general multiple scales ansatz in the case of atmospheric flows allows us to systematically recover a large number of simplified models of theoretical meteorology in a transparent fashion. Of course, the true value of such a general ansatz lies in its potential for describing scale interactions. Thus we concluded this report by summarizing the recent development of a "systematic multi-scale model for the tropics" by Majda and the author.

Acknowledgements. This work was sponsored by the Deutsche Forschungsgemeinschaft, grant KL 611/5, KL 611/6, KL 611/14. The author would like to thank Ann Almgren, Nicola Botta, Julian Hunt, Omar Knio, Andrew Majda, Eileen Mikusky, Claus-Dieter Munz, Antony Owinoh, Vladimir Petukhov, and Stefan Vater, for a wealth of challenging questions, stimulating discussions, and encouraging comments!

\section{REFERENCES}

[1] P.R. Bannon, On the anelastic approximation for a compressible atmosphere. J. Atmosphere Sci. 53 (1996) $3618-3628$.

[2] G.K. Batchelor, The conditions for dynamical similarity of motions of a frictionless perfect gas atmosphere. Quart. J. Roy. Meteorol. Soc. 79 (1953) 224-235.

[3] J.R. Biello and A.J. Majda, A new multiscale model for the madden julian oscillation. J. Atmosphere Sci. 62 (2005) in press.

[4] N. Botta, R. Klein and A. Almgren, Asymptotic analysis of a dry atmosphere. ENUMATH, Jyväskylä, Finland (1999).

[5] C. Bretherton and A. Sobel. The gill model and the weak temperature gradient (wtg) approximation. J. Atmosphere Sci. 60 (2003) 451-460.

[6] J.G. Charney, A note on large-scale motions in the tropics. J. Atmosphere Sci. 20 (1963) $607-609$.

[7] S.B. Dorofeev, V.P. Sidorov, A.E. Dvoinishnikov and W. Breitung, Deflagration to detonation transition in large confined volume of lean hydrogen-air mixtures. Combustion E Flame 104 (1996) 95-110.

[8] D.R. Durran, Improving the anelastic approximation. J. Atmosphere Sci. 46 (1989) 1453-1461. 
[9] A.E. Gill, Some simple solutions for heat-induced tropical circulation. Quart. J. Roy. Meteorol. Soc. 87 (1980) $447-462$.

[10] I.M. Held and B.J. Hoskins, Large-scale eddies and the general circulation of the troposphere. Adv. in Geophysics 28 (1985) $3-31$.

[11] J.C.R. Hunt, K.J. Richards and P.W.M. Brighton, Stably stratified shear flow over low hills. Quart. J. Roy. Meteorol. Soc. 114 (1988) 859-886.

[12] R. Klein, Semi-implicit extension of a godunov-type scheme based on low mach number asymptotics I: One-dimensional flow. J. Comput. Phys. 121 (1995) 213-237.

[13] R. Klein, Asymptotic analyses for atmospheric flows and the construction of asymptotically adaptive numerical methods. ZAMM 80 (2000) 765-777.

[14] R. Klein, An applied mathematical view of theoretical meteorology, in Applied Mathematics Entering the 21st Century; Invited talks from the ICIAM 2003 Congress, SIAM Proceedings in Applied Mathematics 116 (2004).

[15] R. Klein, Multiple scales asymptotics for atmospheric flows, in Proceedings of the 4th European Conference on Mathematics, Stockholm, Sweden (2004).

[16] R. Klein and S. Vater, Mathematical modelling in climate research. Technical report, Freie Universität, Berlin, Germany (2005).

[17] H. Lamb, Hydrodynamics. Dover Publishers, New York (1981).

[18] F. Lipps and R. Hemler, A scale analysis of deep moist convection and some related numerical calculations. J. Atmosphere Sci. 39 (1982) 2192-2210.

[19] F. Lipps and R. Hemler, Another look at the scale analysis of deep moist convection. J. Atmosphere Sci. 42 (1985) 1960-1964.

[20] R. Madden and P. Julian, Description of global cell circulation cells in the tropics with a 40-50 day period. J. Atmosphere Sci. 29 (1972) 1109-1123.

[21] A.J. Majda and J.A. Biello, A multiscale model for tropical intraseasonal oscillations. Proc. Natl. Acad. Sci. USA 101 (2004) $4736-4741$.

[22] A. Majda and R. Klein, Systematic multi-scale models for the tropics. J. Atmosphere Sci. 60 (2003) 393-408.

[23] A. Majda and J. Sethian, The derivation and numerical solution of the equations for zero Mach number combustion. Combust. Sci. Tech. 42 (1985) 185-205.

[24] T. Matsuno, Quasi-geostrophic motions in the equatorial area. J. Meteorol. Soc. Jap. 44 (1966) 25-43.

[25] T.M.J. Newley, H.J. Pearson and J.C.R. Hunt, Stably stratified rotating flow through a group of obstacles. Geophys. Astrophys. Fluid Dynam. 58 (1991) 147-171.

[26] Y. Ogura and N.A. Phillips, Scale analysis of deep moist convection and some related numerical calculations. J. Atmosphere Sci. 19 (1962) 173-179.

[27] J. Oliger and A. Sundstroem, Theoretical and practical aspects of some initial boundary value problems in fluid dynamics. NASA STI/Recon Technical Report N 7725465 (November 1976).

[28] J. Pedlosky, Ed., Geophysical Fluid Dynamics. Springer, Berlin, Heidelberg, New York, 2nd edition (1987).

[29] V. Petoukhov, A. Ganopolski, V. Brovkin, M. Claussen, A. Eliseev, C. Kubatzki and S. Rahmstorf, Climber-2: A climate system model of intermediate complexity. Part I: Model description and performance for the present climate. Climate Dynamics 16 (2000) $1-17$.

[30] A.S. Worlikar and O.M. Knio, Numerical study of oscillatory flow and heat transfer in a loaded thermoacoustic stack. Numer. Heat Transfer 35 (1999) 49-65.

[31] A.S. Worlikar, O.M. Knio and R. Klein, Numerical simulation of a thermoacoustic refrigerator. II: stratified flow around the stack. J. Comput. Fluids 144 (1998) 299-324.

[32] Th. Schneider, N. Botta, R. Klein and K.J. Geratz, Extension of finite volume compressible flow solvers to multi-dimensional, variable density zero Mach number flow. J. Comput. Phys. 155 (1999) 248-286.

[33] Th. Schneider, R. Klein, E. Besnoin and O. Knio, Computational analysis of a thermoacoustic refrigerator, in Proceedings of the joint EAA/ASA meeting (March 1999).

[34] S. Schochet, The mathematical theory of low mach number flows. ESAIM: M2AN 39 (2005) 441-458.

[35] V. Smiljanovski, V. Moser and R. Klein, A capturing-tracking hybrid scheme for deflagration discontinuities. J. Combustion Theory Modeling 1 (1997) 183-215.

[36] A.A. Sobel, J. Nilsson and L. Polvani, The weak temperature gradient approximation and balanced tropical moisture waves. J. Atmosphere Sci. 58 (2001) 3650-3665.

[37] P.J. Webster, Response of the tropical atmosphere to local steady forcing. Monthly Weather Review 100 (1972) 518-541.

[38] A.A. White, A view of the equations of meteorological dynamics and various approximations, in Large Scale Atmosphere-Ocean Dynamics I: Analytical Methods and Numerical Models. J. Norbury and I. Roulstone, Eds., Cambridge University Press (2002).

[39] G.B. Whitham, Linear and Non Linear Waves. John Wiley (1974).

[40] R.K. Zeytounian, Meteorological Fluid Dynamics. Number m5 in Lecture Notes in Physics. Springer, Heidelberg, Berlin, New York (1991). 